\title{
Zur Theorie des Wachsthums und der Befruchtung.
}

\author{
Von \\ Julius Bernstein, \\ Halle a. S. \\ Mit 1 Figur im Text. \\ Eingegangen am 16. September 1898.
}

\section{Vorbemerkung.}

Die vielfachen theoretischen Erörterungen der letzten Zeit im Gebiete der Entwickelungslehre, welche bisher fast aussebließlich von Morphologen herrühren, haben mich dazu angeregt, im Nachstehenden diejenigen Gesichtspunkte hervorzuheben, welche mir von physiologischem oder besser gesagt von allgemein naturwissenschaftlichem Standpunkte für die Auffassung der Entwickelungsvorgänge von Bedeutung zu sein scheinen. Bei dem vielfachen Widerstreit der Meinungen unter den Morphologen dürfte vielleicht eine Stimme aus dem angrenzenden Lager der Physiologie nicht unwillkommen sein.

An die Spitze meiner Betrachtung möehte ich den Satz stellen, dass zur Begriindung einer »cansalen«, d. h. »mechanistischen " Entwickelungslehre, wie sie von Roux als "Entwickelungsmechanik " aufgefasst wird, neben der Formbildung die "Energetik « der stattfindenden Vorgänge in Betracht gezogen werden muss. So wenig wir auch von der Natur und dem Zusammenhang der dabei mitwirkenden Kräfte Kenntnis haben, so lassen sich doch gewisse Principien der modernen »Energielehre auf die Entwickelungsvorgänge anwenden, so weit sie auch auf physiologische Thätigkeiten mit Erfolg angewendet sind. 


\section{Wachsthum und Befruchtung.}

Den Stoffwechsel in der lebenden Substanz vorausgesetzt, ist »Wachsthum « die Grundbedingung jeder Formentwickelung oder Formveränderung, also anch der Fortpflanzung. Die Fähigkeit eines Organismus, sich fortzupflanzen, hängt daher von seiner »Wachsthumsenergie* im Allgemeinen und Besonderen ab. Unter $»$ Wachsthumsenergie« sei die Gesammtheit der physikalischen und ehemischen Energien verstanden, welche das Wachsen zu Stande bringen.

Die Vorgänge des Wachsthums bestehen in allen Organismen, pflanzlichen wie thierischen, von den einfachsten bis zu den komplicirtesten, nach dem Princip der Erhaltung der Energie in einer Ansammlung potentieller Energien, sei es, dass diese aus der kinetischen Energie des Lichtes und der Wärme oder aus aufgenommenen chemischen Energien der Nährstoffe und des Sanerstoffes herriuhren. Diesen Vorgang hat man auch »Assimilirung « genannt.

Denken wir uns nun die einfachste lebende Substanz als homogene, formlose Materie. Denn unbekümmert um die Streitfrage, ob eine solche existirt oder existirt hat, können wir einer solchen Materie die Fähigkeit des Wachsthums unter geeigneten Existenzbedingungen zuertheilen. Nehmen wir an, dass sie ins Unbegrenzte wachse, so fallen Wachsthum und Fortpflanzung in ihrer Erscheinung: vollständig zusammen. Eine Zertheilung dieser wachsenden Masse durch äußere Einwirkung in beliebige Theile ändert nichts an diesem Vorgange, würde aber durch Vergrößerung der Oberfläche in Folge schnellerer Aufnahme von Stoffen eine Beschleunigung des Wachsthums mit sich bringen ${ }^{1}$ ).

Das Wachsen würde nun bei konstanten äuBeren Bedingungen in dieser Weise ins Unbegrenzte fortgesetzt werden, wenn sich demselben nicht »Widerstände《 oder »Hemmungen entgegenstellten. Dass bei einem jeden Organismus, abgesehen von äußeren Widerständen, »innere Hemmungen « des Wachsthums auftreten, lässt sich sowohl aus der Begrenzung des Wachsthums als ans der der Lebensdauer schließen. In letzterem Falle sind die hemmenden Kräfte solche, welche den Stoffwechsel iiberhaupt aufheben, im ersteren Falle solche, welche nur die Energien des Wachsthums vernichten. Deuten wir diese Energien als chemische, so könnten die Hemmungen durch Erzengung gewisser Stoffwechselprodukte hervorgebracht werden.

1) Siehe auch: Bernsteis, Über die Kräfte der lebenden Materie. Osterprogramm d. Univ. Halle. 1880. pag. 17. 
Bekannt ist es z. B., dass die Produkte der Gärungen . hemmend anf die Entwickelung der Gärungspilze einwirken etc.

Wir statuiren demnach in Bezug auf das Wachsthum im Allgemeinen zwei Arten von Kräften, erstens die treibenden und zweitens die hemmenden Kräfte. Es wird gut sein, bei der Komplieirtheit der Vorgänge sich diese beiden Arten von Kräften zunächst in allgemeiner Form vorzustellen.

Die Vorstellnng treibender (resp. erregender) und hemmender Kräfte hat sich in der Physiologie des Nerven- und Muskelsystems als sehr zweckdienlich erwiesen. Zur Erklärung der Thätigkeit rhythmisch arbeitender Centren, z. B. des Athem- und Herzcentrums, hat man angenommen, dass eine treibende Kraft in der in dem Organ durch die Ernährung sich ansammelnden potentiellen Energie enthalten ist und dass eine hemmende Kraft sich der Umwandlung in kinetische Energie entgegenstellt, dass aber vermöge einer eigenen inneren Mechanik (Molekularmechanik) eine rhythmische Durchbrechung der Hemmung durch die treibende Kraft stattfinde [v. BEzOLD ${ }^{1}$ ), Rosent'HaL $\left.{ }^{2}\right)$ ]. Alle Vorgänge der Reizung und der Veränderung der Reizbarkeit lassen sich nach PFLüger ${ }^{3}$ ) auf die Vorstellung von zwei derartigen Kräften (Molekularspannungen und Molekularhemmungen) zurïckführen, wenn wir die von außen zugeführten Reize als auslösende Kräfte betrachten. Es scheint mir daher gerechtfertigt, eine entsprechende Vorstellung auch auf die Vorgänge des Wachsthums und der Fortpflanzung zu übertragen.

Kehren wir nun zur Betrachtung der formlosen lebenden Materie zurück, so würde unter der Annahme von entstehenden Hemmungen auch ihr Wachsthum nicht unbegrenzt sein können. Doch mögen in einer von uns angenommenen lebenden Urmaterie diese Hemmungen außerordentlich langsam entstanden sein, entsprechend den sehr geringfügigen Lebensthätigkeiten dieser Materie. Sobald aber durch den Wechsel der Lebensbedingungen eine Variabilität in dieser Materie auftrat und damit zugleich der Kampf ums Dasein eröffnet wurde, musste sich das Bild ändern. Die besser oder zweckmäßiger konstituirten Massen mit erhöhter Lebensthätigkeit verdrängten die schlechter konstituirten, zugleich aber nahmen auch mit der Beschleunigung des Wachsthums und der Lebensthätigkeiten die hemmenden Kräfte schneller zu. Daher konnte eine weitere Entwicke-

1) Untersuchungen über die Innervation des Herzens. 1863.

2) Die Athembewegungen. 1862.

3) Untersuchungen über die Physiologie des Elektrotonas. 1859. 
lung zu höheren und zweckmäßigeren Formen nur dann stattfinden, wenn durch irgend einen Vorgang das Wachsen der hemmenden Kräfte beschränkt wurde. Dieser Vorgang ist, wie ich vermuthe, in seiner einfachsten Gestalt die Konjugation, aus welcher sich die Befruchtung entwickelt hat.

Denken wir uns demnach eine homogene lebende Urmaterie, so wird durch die Verschicdenheit änBerer Einwirkungen anf verschiedene Massen derselben eine wenn auch Anfangs nur geringe Variation derselben hervorgebracht werden können. Ich denke mir also, dass in dieser Urmaterie die äußeren Einwirkungen, die niemals ganz gleich sein können, auch die primäre Ursache der »Variabilität « in DARwin'sehem Sinne gewesen seien. In jedem Massentheilchen entsteht aber eine der treibenden Kraft des Wachsthums entgegengesetzte hemmende Kraft; und wenn diese eine gewisse Größe erreicht hat, so wuirde sie dem weiteren Wachsthum ein Ziel setzen. Nun musste es aber der "Zufall fügen, welcher in DarwiNschem Sinne als *Nothwendigkeit« zu betrachten ist, dass zwei Massentheilchen von mehr oder weniger variirter Beschaffenheit mit einander in Berithrung kamen und sich vereinigten. Und diese Vereinigung, welche unter den jetzt lebenden einfachsten Organismen noch als "Konjugation « erscheint, musste sich als zweckmäßig erwiesen haben, indem sie die Wachsthumsenergie erhöhte. Ein soleher Erfolg kann nun nach unserer Theorie durch Beseitigung oder Schwächung der hemmenden Kräfte herbeigeführt sein. Man stelle sich gan $z$ allgemein vor, dass in dem Molekül $a_{1}$ des ersten Massentheilchens sich die hemmende Kraft $l_{1}$ bildete, und in dem Molekiil $a_{2}$ des zweiten Massentheilchens die hemmende Kraft $l_{2}$, wobei sowohl $a_{1}$ und $a_{2}$ als anch $h_{1}$ und $h_{2}$ von etwas verschiedener Natur (resp. Qualität) sein mögen; entsteht num durch die Vereinigung. der Molekiile $a_{1}$ und $a_{2}$ ein drittes Molekiil $a_{3}$ (oder zwei und mehrfache derselben) von wiederum etwas anderer Beschafienheit, so wïrden die hemmenden Kräfte $h_{1}$ und $h_{2}$ anf dieses nicht oder weniger einwirken können, als sie auf $a_{1}$ und $a_{2}$ eingewirkt haben, da ibre Natur nicht der Natur von $a_{3}$ entspricht. Dies wird immer unter der Bedingung der Fall sein, dass die treibenden Kräfte durch ihre Vereinigung sich der Einwirkung der hemmenden Kräfte mehr oder weniger entziehen, oder dadurch, dass die hemmenden Kräfte einander entgegenwirken. Es wïrde dies aber nicht der Fall sein, wenn etwa die Kräfte $h_{1}$ und $h_{2}$ sich zu einer dem Molekül $a_{3}$ entsprechenden hemmenden Kraft $h_{3}$ vereinigten. Es möge dies durch 
einige Beispiele erläutert werden. Zuerst ein rein mechanisches. Man denke sich zwei durch bestimmte treibende Kräfte, welche beständig von aufen wirken, in Rotation versetzte Räder, deren Achsen an einem senkrechten Faden hängen, in gewisser Entfernung von einander, so dass ibre Ebenen zusammenfallen. An den beiden von einander abgewendeten Punkten ihrer Peripherie befinde sich an jedem Rade eine Bremse, welche die Bewegung verzögert und deren Reibung mit der Zeit zunehme. Die Rotation des einen Rades möge
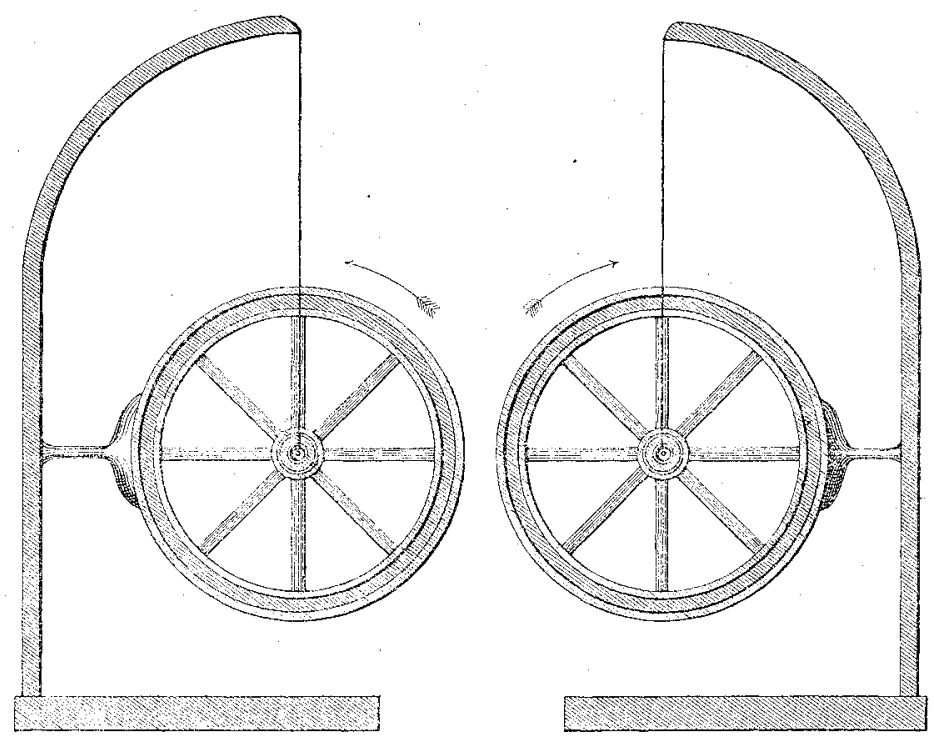

nach der einen, die des anderen nach der anderen Seite serichtet sein, was die Variation der Molekïle $a_{1}$ und $a_{2}$ vorstellen möge. Man bringe sie nun einander näher und es möge bei geringerer Entfernung dnrch eine gewisse durch ihre Einwirkung gegebene Kraft (z. B. Massenanziehung) eine gegenseitige Anziehung stattfinden, so werden sich beide von ihren Bremsen entfernen nnd die Geschwindigkeit ihrer nun gemeinsamen Rotationen wird wieder zunehmen (siehe beistehende Figur).

Es kommt hierbei selbstverständlich auf die Bedingungen des Mechanismus wesentlich an. Würden die Achsen von in gleichen Richtungen rotirenden Rädern in einer geraden Linie liegen, so würde bei einer Vereinigung der Achsen das Verhältnis der summirten hemmenden and treibenden Kräfte dasselbe bleiben wie vorher. Das 
würde dann einer Vereinigung ganz gleichartiger lebender Massentheilchen entsprechen, welche keinen Vortheil haben könnte.

Ein anderes mechanisch-chemisches Beispiel seien zwei Tropfen Flüssigkeit, in denen ein System kleiner Körperchen durch innere Kräfte in Bewegung erhalten werde (z. B. BRown'sche Molekularbewegung). Der Bewegung leistet als hemmende Kraft die innere Reibung der Flüssigkeit Widerstand, und es möge dureh eine in beiden differente Abseheidung der Körperchen der Reibungskoefficient der Flüssigkeit immer mehr zunehmen, so würde in beiden mit der Zeit ein Stillstand der Bewegung eintreten. Mischen sich nun die beiden Tropfen mit einander, so möge durch die chemische Verbindung der Abscheidungen der Reibungskoefficient vermindert werden, so dass die Bewegung von Neuem eingeleitet wird.

Auch kann man eine rein chemische Deutung des Vorganges in allgemeiner Form geben. Es sei das Molekül der beiden Massentheilchen so gebant, dass an einen Atomkomplex $M_{1}$ und $M_{2}$ eine Seitenkette $x_{1}$ und $x_{2}$ sich anlegt, welche allmählich wachsend die Assimilirungsfähigkeit der Molekuile hemmt. Bei der Vereinigung beider Moleküle gehe eine Spaltung nach der Formel $\left.{ }^{1}\right):\left(M_{1}-x_{1}\right)+\left(M_{2}-x_{2}\right)$ $=\left(M_{1}-M_{2}\right)+\left(x_{1}-x_{2}\right)$ vor sich, und nach Ausstoßung von $\left(x_{1}-x_{2}\right)$ entstehe nach der Formel $\left(M_{1}-M_{2}\right)=2 \cdot M_{3}$ ein neues mit verstärkter Assimilationsfähigkeit begabtes Molekül $\boldsymbol{M}_{3}$.

Es möchte hinzugefügt werden, dass man sich das Wachsen der lebenden Substanz entsprechend der Pfü̈ger'schen Theorie so denken kann, dass jedes Molekül etwa durch Polymerisirung so weit wächst, bis es in zwei Moleküle zerfällt.

Bei vielen Infusorien findet der einfachste Vorgang der Befruchtung, die Konjugation, nur von Zeit zu Zeit statt ${ }^{2}$. Durch mehrere Generationen hindurch kann in ungestörtem Wachsthum die Vermehrung durch Theilung vor sich gehen. Aber wenn man die Kulturen sorgfältig von einander trennt, tritt allmählich eine Wachsthumshemmung ein. Bei der Vermischung der Kulturen aber findet durch Konjugation eine Wiederbelebung des Wachsthums statt; und worin kann dieser Vorgang anders bestehen als in einer gegenseitigen Aufhebung der hemmenden Kräfte. Denn eine Neuerzeugung treibender Kräfte aus zwei Körpern, in denen diese Kräfte nicht mehr

1) Die Zeichen - stellen chemische Affinitäten vor.

2) Maupas, Théorie de la sexualité des Infusoires eiliés. Compt. rend. T. 105 . pag. $356-358$. 
vorhanden waren, ist nach dem Gesetz von der Erhaltung der Energie nicht denkbar.

Ist nun erst einmal bei den niedersten Organismen dieser mächtige Stimulus des Wachsthums, die Konjugation, wirksam geworden, dann ergiebt sich in der phylogenetischen Entwickelung durch die Anpassung im Kampfe ums Dasein und durch die Vererbung die weitere Vervollkommnung des Befruchtungsvorganges als eine Nothwendigkeit. Je differenter die sich konjugirenden Gebilde geworden sind, wobei die Fähigkeit der Vereinigung erhalten werden musste, desto ergiebiger wurde die Wachsthumsenergie. So schritt die Differenzirung der Keime mit der phylogenetischen Entwickelung vorwärts und so kam es schließlich nach dem Principe der Arbeitstheilung zu der Entstehung des männlichen und weiblichen Keimes und der Geschlechter. Die Ei- und die Samenzelle einer Species sind daher als Gebilde anzusehen, welche eine möglichst große Differenz der Organisation erlangt haben, und zwar mit Erhaltung der Fähigkeit, sich zu einem gemeinsamen Ganzen zu vereinigen. Während alle anderen Zellen des Organismus eine Wachsthums- und Entwickelungsgrenze durch Zunahme der hemmenden Kräfte erreichen, vermögen Ei- und Samenzelle durch ihre Vereinigung die hemmenden Kräfte wiederum zu eliminiren. Dass eine Differenzirung der Keime nach dem Princip der Arbeitstheilung in ihnen stattgefunden, indem das Samenkörperehen die Funktionen der Bewegung und der Reizbarkeit, die Eizelle die Funktion der Ernährung durch Ansammlung von Nährmaterial in höherem Grade ausgebildet hat, ist schon von HAEckeL und Hertwig erkannt worden. Diese an sich vortheilhafte Arbeitstheilung hat aber zugleich den Sinn, dass die sich vereinigenden Moleküle eine möglichst versehiedene Konstitution besitzen, so weit dies zur Eliminirung der hemmenden Kräfte erforderlich ist.

Es möge noch bemerkt werden, dass mit der Erhöhung der Wachsthumsenergie durch Befruchtung auch die Möglichkeit und Mannigfaltigkeit der Variabilität gefördert werden mussten. So lange beim Wachsen einer lebenden Materie eine Konjugation nicht statt hatte, war die Variabilität allein das Produkt der äuBeren Einwirkungen auf jeden besonderen Theil der Materie für sich. Sobald aber Konjugation resp. Befiuchtung eingetreten war, nahm auch die Fähigkeit der Variabilität mit der Wachsthumsenergie zu und es addirten sich die Möglichkeiten der Variabilität aus beiden Komponenten der Vereinigung zu einander. Die bewirkenden Ursachen der Variabilität können aber immer nur die äußeren Kräfte sein, und 
zwar sowohl die auf die Keime als auch die auf den wachsenden Organismus einwirkenden Kräfte.

Es kann aus dem Vorhandensein einer Fortpflanzung durch Knospung und Parthenogenesis ein Einwand gegen die aufgestellte Theorie nicht hergeleitet werden. Die Parthenogenesis ist immer nur auf eine oder wenige Generationen beschränkt; die Fortplanzung durch Knospung kommt nur bei Pflanzen und den niederen thicrischen Organismen vor und beweist nur, dass eine Fortsetzung des Wachsthums ohne Befruchtung bis zu einer gewissen Entwickelungsstufe möglich ist, aber nicht zu höheren Formen führen kann. Außerdem kommen bei knospenden Organismen anch Konjugationen und Befruchtungen vor, welche sich als vortheithaft erweisen.

Fasse ich daher die aufgestellte Theorie in wenige Worte zusammen, so lantet sie folgendermaßen: »Die treibenden Kräfte, welche das Wachsthum bedingen und deren Energie von außen zugeführt wird (Licht, Wärme, chemische Energie), arbeiten in der organisirten Materie gegen hemmende Kräfte, welche den Molekülen der Materie zukommen ${ }^{1}$ ). Letztere nehmen in Folge der chemischen und physikalisehen Änderungen der Materie mit der Dauer des Wachsthumsprocesses an Intensität mehr und mehr zu, so dass sie schließlich den vollkommenen Stillstand alles Wachsens herbeiführen (Tod des Individuums). Jede Art von Befruchtung hat dagegen die unmittelbare Folge, dass vermöge einer Einwirkung mehr oder weniger differenter Materien eine gegenseitige Schwächung oder Aufhebung der hemmenden Kräfte stattfindet, so dass die treibenden Kräfte von Nenem anbeben, ihre volle Wirksamkeit zu entfalten."

\section{Morphologische Deutung und mechanische Analyse.}

Es könnte scheinen, als ob die vorhergehenden Betrachtungen im Sinne einer mechanischen Anschauung der Entwickelungsvorgänge zu den bisher von den Morphologen aufgestellten Ansichten in einem Gegensatze stünden. Dies ist bei näherer Prüfung keineswegs im Princip der Fall. Ja es sind sogar beide Arten der Betrachtung zu einer befriedigenden Lösung der vorliegenden Aufgaben durch gegenseitige Ergänzung durchaus erforderlich. Um dies klar zu machen, ist es zweekmäßig, auf die einfacheren Aufgaben der analytischen

1) Das einfachste mechanische Analogon derselben ist die Reibung bei der Bewegung von Massen gegen einander. 
Mechanik zurückzugreifen. Wenn die Bewegungen eines Systems von materiellen Punkten und die Konfigurationen desselben in jedem Zeitpunkte bestimmt werden sollen, und wenn die Punkte desselben nicht frei, sondern gewissen Bedingungen in ihrer Bewegung unterworfen sind, so müssen diese Bedingungen durch gegebene Gleichungen ansgedrückt werden, wenn man die Einwirkung gewisser derart wirkender Kräfte ermitteln will. Ist z. B. ein Punkt gezwungen, sich nur auf einer Kugelschale zu bewegen, wie auch die änßeren Kräfte auf ihn einwirken mögen, so muss die Gleichung der Kugelfläche mit den Gleichungen seiner Lage und Bewegung vereinbar sein. Die Gleichung der Kugel nennt man in diesem Falle die „Bedingungsgleichung" der Bewegung. Sind für alle Punkte eines Systems diese Bedingungsgleichungen gegeben und ebenso die von außen einwirkenden Kräfte, so ist das Problem nach Lagrange im Princip lösbar.

Die "morphologischen Deutungen « eines biologischen Vorganges verhalten sich nun zu der "eausalen mechanischen Analyse " desselben im Großen und Ganzen wie die Aufstellung jener "Bedingungsgleichungen « zur »analytischen Berechnung«. Die morphologische Deutung des Problems giebt uns gewissermaßen das Endziel an, nach welchem unter dem Anschein eines Zweckes ein Entwickelungsund Wachsthumsprocess hinstrebt, und darin sind die "Bedingungen" enthalten, unter welchen der ganze Process abläuft. Freilich sind wir weit davon entfernt, diese durch Gleichungen ausdruicken zu können. Aber auch wenn wir dies könnten, wäre damit noch keineswegs eine causale, mechanistische Erklärung des Vorganges gefunden. Es fehlte vielmehr die Aufstellung der »treibenden Kräfte«, welche an dem biologischen System den festgesetzten »Bedingungen " gemäß Wirkungen ausuiben.

Die morphologische Dentung eines biologischen Problems kann sich selbstrerständlich nicht auf bloße Bedingungen der Konfiguration beschränken, wie dies bei mechanischen Problemen der Fall ist, sondern muss biologische Gesichtspunkte zu Hilfe nehmen. Als ein Beispiel einer solchen morphologischen Deutung im Großen und Allgemeinen haben wir die DarwiN'sche Theorie zu betrachten. Nach dieser Theorie soll die Formentwickelung immer eine solche sein, dass die für den Kampf ums Dasein zweckmäßig konstituirten Formen bestehen bleiben, während die unzweckmäßigen Abänderungen schon in ihren ersten Anfängen eliminirt werden. Könnten wir diese Bedingung der phylogenetischen Entwickelung durch Gleichungen ausdrücken, so hätten wir die erste Grundlage zur folgenden mechani- 
schen Analyse jener geschaffen. Wir müssen uns indessen damit begnügen, aus der Vergleichung des Thatsachenmaterials zusammenfassende Gesichtspunkte zu kombiniren, deren Werth nach dem Grade ihrer Wahrscheinlichkeit im allgemein naturwissenschaftlichen Sinne geprüft werden muss. Das ist die Aufgabe der auf vergleichender Anatomie und Entwickelungsgeschichte sich aufbauenden "Descendenzlehre", wie sie Darwin, HaEckel und Andere begründet haben. Einen wichtigen Gesichtspunkt dieser Art von allgemeiner Bedeutung bildet z. B. das von HAECKEL benannte »biogenetische Grundgesetz«. Es ist klar, dass die Kenntnis dieser Bedingungen der Entwickelung, welche den ersten Theil des Problems bildet, nur eine sehr unvollkommene ist und bleiben wird. Doch setzen wir in einem Falle die Lösung dieses Theils als richtig gegeben voraus, so würde der zweite Theil des Problems, »die mechanische Analyse ", doch noch unendliche Schwierigkeiten darbieten. Dieser zweite Theil des Problems fällt aber mit dem zusammen, was nach den Definitionen von Roux als „Entwickelungsmechanik " bezeichnet wird. Zur Lösung dieser Aufgabe ist es weiterhin erforderlich, über die Art der auf das biologische System von außen her einwirkenden Kräfte die geeigneten Hypothesen aufzustellen. Es ist ebenso einleuchtend, dass auch die Lösung dieses Theils des Problems immer nur eine sehr unvollständige ond lückenhafte sein kann !).

Kehren wir nach diesen allgemeinen Betrachtungen wieder zu dem speciellen Thema dieser Mittheilung zurïck, so können wir nach unserer Darlegung das besprochene Problem folgendermaßen zerlegen:

Die »morphologische Deutung « der Befruchtung besteht in der Annahme, dass wir eine durch Stoffwechsel wachsende Materie haben, dass diese nach dem Princip der Variabilität sich abgeändert und in kleinere Elemente (Zellen) von variabler Beschaffenheit zertheilt habe, und dass es für das Wachsthum rortheilhaft gewesen sei, wenn solche Elemente von möglichst divergenter Beschaffenheit sich mit einander vereinigt haben. In diesen Annahmen würden die »Bedingungsgleichungen * des Problems enthalten sein. Die »mechanische

1) Die Bedingungsgleichungen eines biologischen Problems müssen immer komplicirter werden, je weiter die phylogenetische Entwickelung vorschreitet, indem die Resultate der mechanischen Analyse des unmittelbar vorhergehenden Problems in die Bedingungen des nachfolgenden eingehen, worin das Moment der $\curvearrowright$ Vererbnng* gegeben sein wïrde. 
Analyse des Problems wurde in Angriff genommen, indem wir die Hypothese von der Existenz »treibender" und »hemmender Kräfte hinzufigten, welche in gewisser Weise in der Materie wirken sollen. Diese Lösung konnte selbstverständlich nur eine ganz allgemeine Form annehmen. Wir sind weit davon entfernt, sie auf alle Details der Zelltheilung in Anwendung zu bringen. Und doch scheint es mir nicht ganz aussichtslos, von dem eingenommenen Standpunkte aus Einzelheiten der morphologischen Wandlungen der Zellen beim Wachsen und Vermehren zu beleuchten. Forschung und Kritik mögen entscheiden, ob die gemachten Hypothesen einen Fortschritt der Erkenntnis herbeifübren können.

Zum Schluss bitte ich um Entschuldigung, wenn ich in dieser Mittheilung Manches aus der amfangreichen morphologischen Litteratur, was mit meiner Theorie einige Ähnlichkeit haben möge, nicht berücksichtigt haben sollte. Es ist unter den heutigen Verhältnissen nicht mehr möglich, anch noch die Litteratur einer angrenzenden Wissenschaft nur annähernd zu bewältigen. Wenn ich daher Einigen etwas nicht ganz Neues gesagt haben sollte, so habe ich mich doch bemïht, den Kern der Sache in einer neuen Form, in der exakteren Sprache der Mechanik, darzustellen. 